\title{
A Fast pH-Switchable and Self-Healing Supramolecular Hydrogel Carrier for Guided, Local Catheter Injection in the Infarcted Myocardium
}

\author{
Maartje M. C. Bastings, Stefan Koudstaal, Roxanne E. Kieltyka, Yoko Nakano,
} A. C. H. Pape, Dries A. M. Feyen, Frebus J. van Slochteren, Pieter A. Doevendans, Joost P. G. Sluijter, E. W. Meijer, Steven A. J. Chamuleau,* and Patricia Y. W. Dankers*

Minimally invasive intervention strategies after myocardial infarction use state-of-the-art catheter systems that are able to combine mapping of the infarcted area with precise, local injection of drugs. To this end, catheter delivery of drugs that are not immediately pumped out of the heart is still challenging, and requires a carrier matrix that in the solution state can be injected through a long catheter, and instantaneously gelates at the site of injection. To address this unmet need, a pH-switchable supramolecular hydrogel is developed. The supramolecular hydrogel is switched into a liquid at $\mathrm{pH}>8.5$, with a viscosity low enough to enable passage through a $1-\mathrm{m}$ long catheter while rapidly forming a hydrogel in contact with tissue. The hydrogel has self-healing properties taking care of adjustment to the injection site. Growth factors are delivered from the hydrogel thereby clearly showing a reduction of infarct scar in a pig myocardial infarction model.

\section{Introduction}

Coronary artery disease is a progressive disease that can be held responsible for over seven million deaths worldwide each year, and the increased aging of the population will even yield a further rise in mortality and morbidity..$^{[1]}$ New strategies are aiming at the prevention of the progression of postmyocardial infarction toward heart failure. Catheter-based drug delivery injection approaches ${ }^{[2,3]}$ are substantially less invasive than for example surgical implantation of in vitro engineered tissues, ${ }^{[4]}$ patches, ${ }^{[5,6]}$ or drug delivery carriers. ${ }^{[7]}$ Therefore, catheter-injection strategies are the method of choice with regard to clinical applicability. State-of-the-art is the NOGA catheter that enables precise control over the injection location via a special mapping system. ${ }^{[8]}$ A 3D electromechanical image of the myocardium can be obtained using an ultralow magnetic-field energy source and a sensor-tipped catheter to locate the catheter position. This mapping allows for the accurate identification of normal and infarcted myocardium, and in this way, enables excellent spatial control over the injection of drugs. Generally, the injected drugs are substantially fast removed from the pulsatile heart when not delivered via a solid or gelated carrier material. Therefore, the
Dr. M. M. C. Bastings, [i๋] Dr. R. E. Kieltyka, Dr. Y. Nakano,

A. C. H. Pape, Prof. E. W. Meijer, Dr. P. Y. W. Dankers

Institute for Complex Molecular Systems

Eindhoven University of Technology

PO Box 513, 5600 MB Eindhoven, The Netherlands

E-mail: p.y.w.dankers@tue.nl

Dr. M. M. C. Bastings, Dr. R. E. Kieltyka, Dr. Y. Nakano,

A. C. H. Pape, Prof. E. W. Meijer, Dr. P. Y. W. Dankers

Laboratory of Chemical Biology

Eindhoven University of Technology

PO Box 513, 5600 MB Eindhoven, The Netherlands

S. Koudstaal, ${ }^{[+]}$D. A. M. Feyen, F. J. van Slochteren, Prof. P. A. Doevendans,

Dr. J. P. G. Sluijter, Dr. S. A. J. Chamuleau

Department of Cardiology

Division Heart and Lungs

University Medical Center Utrecht

PO Box 85.500, 3508 GA Utrecht, The Netherlands

E-mail:s.a.j.chamuleau@umcutrecht.nl
S. Koudstaal, Prof. Dr. P. A. Doevendans, Dr. J. P. G. Sluijter, Dr. S. A. J. Chamuleau Interuniversity Cardiology Institute of the Netherlands (ICIN), PO Box 19258, 3501 DG Utrecht The Netherlands

Prof. Dr. E. W. Meijer

Laboratory of Macromolecular and Organic Chemistry Eindhoven University of Technology

PO Box 513, 5600 MB Eindhoven, The Netherlands

[iे]These authors contributed equally to this work.

DOI: 10.1002/adhm.201300076 
development of carrier materials that can be injected through the extremely long and narrow lumen of such catheters, and instantaneously gelate in contact with the myocardial tissue, is prerequisite. The dimensions of the catheters, combined with the dynamic beating environment of the heart, require a material with Janus-like material properties; i.e., low viscosity to flow through the long, narrow lumen of the catheter, and high stability to form a local drug delivery reservoir and provide mechanical support in the tissue after injection. This dual character is proposed to be obtained using a stimuli-responsive, switchable hydrogel. Furthermore, ideally, this hydrogel shows self-healing properties within the high shear environment of the contracting heart muscle.

Natural hydrogels for myocardial injection therapies that have been investigated include fibrin, ${ }^{[9]}$ collagen, ${ }^{[10]}$ alginate, ${ }^{[11]}$ Matrigel, ${ }^{[12]}$ hyaluronic acid,[13] and chitosan. ${ }^{[14]}$ These hydrogels can potentially be delivered in combination with cells or drugs, however, they are derived from a natural source and therefore show batch-to-batch differences. Furthermore, their switching behavior can be poorly controlled. As a consequence, although injection of these hydrogels via a syringe was accomplished relatively easily, the translation to catheter-based delivery has yet remained an unmet challenge. Recently, decellularized porcine extracellular matrix (ECM)-derived hydrogels have been reported as catheter-injectable scaffolds for cardiac regeneration. ${ }^{[15]}$ However, these ECM-derived hydrogels did not show very fast gelation upon injection, and their compatibility with clinical use will be poor because of their animal origin. We propose that synthetic materials fulfill the extensive list of requirements, amongst others that they do not show batch-tobatch differences, they are more easy to switch from sol-gel, and they are not animal, bacteria, or carcinoma derived. ${ }^{[16]}$ Several synthetic systems have been explored for cardiac injection today, including self-assembling peptides ${ }^{[17]}$ and synthetic hydrogels that are formed after injection via in situ chemical or physical cross-linking, ${ }^{[18]}$ photo-induced polymerization, ${ }^{[19]}$ self-assembly, ${ }^{[20]}$ or thermal switching. ${ }^{[21]}$ Again, although easy syringe injection can be obtained with these systems, catheter compatibility remains difficult and has not been shown.

Differences in $\mathrm{pH}$ form interesting parameters to induce switchable behavior in material properties. Since variations of $\mathrm{pH}$ naturally occur in the human body, exploiting these for hydrogel formation is appealing. Various $\mathrm{pH}$-responsive systems appear throughout literature. Importantly, the vast amount of $\mathrm{pH}$-responsive systems are used to enable controlled release of hydrogel payload rather than used to persue a switch in material properties thereby controlling catheter-guided injection. ${ }^{[22]}$

Synthetic supramolecular hydrogelators ${ }^{[23-29]}$ that are held together by directed, noncovalent interactions are proposed to allow for full control of their sol-gel switching behavior under mild conditions using the dynamic nature of the supramolecular interactions. When exploited to the fullest, supramolecular switchable hydrogels systems might be the solution for future clinical catheter-delivery therapies. Therefore, our approach to a synthetic, catheter-injectable hydrogel uses the fourfold hydrogen bonding supramolecular ureido-pyrimidinone (UPy) units ${ }^{[30]}$ coupled via alkyl-urea spacers to $10 \mathrm{k}$ or 20k poly(ethylene glycol) (PEG) chains (Figure 1A). ${ }^{[31,32]}$ These UPy-modified PEG hydrogels form fibers in aqueous solution that are able to cross-link forming transient supramolecular networks. Here, we show that this unique UPy-transient network is $\mathrm{pH}$ responsive, which enables a sol-to-gel switch in a subtle $\mathrm{pH}$ range, and therefore is compatible with the NOGA catheter system. The natural $\mathrm{pH}$ of the tissue instantaneously transforms the injected solution into a drug-loaded hydrogel reservoir. Local in vivo delivery of MRI contrast agents, and active growth factors (GF) hepatocyte growth factor (HGF) and insulin-like growth factor-1 (IGF-1) is demonstrated in a large animal model of ischemic heart disease.

\section{Results and Discussion}

\subsection{Sol-to-Gel pH-Switching Behavior}

Our supramolecular UPy-hydrogel can be made fluid at basic $\mathrm{pH}$, with a threshold at $\mathrm{pH} 8.5$, and reversibly transferred back into a gel state at neutral $\mathrm{pH}$ (Figure 1B). This allows the basic polymer solution to be injected in a solution of neutral $\mathrm{pH}$ (Figure 1C). Almost instantaneous gelling of the polymer material was observed upon the experienced $\mathrm{pH}$ drop. Rheology was performed to investigate the material properties of the hydrogel before and after $\mathrm{pH}$ switching (Figure 1D) and demonstrated that the mechanical properties of the restored hydrogel after $\mathrm{pH}$ switching are identical to the gel before switching. Furthermore, the storage modulus of the $10 \mathrm{wt} \%$ UPy-10k gel matches the mechanical stiffness of the natural cardiac tissue, e.g., $24 \mathrm{kPa}$ for the UPy-hydrogel, versus $26 \mathrm{kPa}$ for adult rat heart muscle ${ }^{[33]}$ (Figure 1D). In addition, the UPy-hydrogels show self-healing behavior (Figure 1F). UPy-hydrogels behave liquid-like at larger deformations $\left(G^{\prime}<G^{\prime \prime}\right)$, but recover within minutes when the deformation is removed (Figure $1 \mathrm{~F}$ ). Viscosity measurements on the basic UPy-10k polymer solution show a viscosity of $0.8 \mathrm{~Pa} \mathrm{~s}$ (Figure 1E), which is very low for a polymer solution and in the same order of magnitude of a solution of plain PEG (without UPy-moieties). ${ }^{[34]}$ After neutralization of the sample, immediately a gel-like sample is formed, confirming the macroscopic observations (Figure 1C). This makes our UPy-hydrogelator an ideal catheter-injectable material. Since the sol-gel transition is fast, we propose that the $\mathrm{pH}$ switchability is caused by breaking of the cross-links between the fibers that form the transient network instead of complete disassembly of the fibers. This results in the fast switching behavior shown. Cartoons are shown to clarify the assembly (and disassembly) process (Figure 2A-D). 1D UPy-stacks in water are formed by UPy dimerization and stacking by additional lateral hydrogen bonding provided by the urea-moieties surrounding the alkyl spacers (that form a hydrophobic pocket) (Figure 2A). These stacks cluster together to form a hierarchical fiber (Figure 2A and B), which entangles and cross-links into a transient network, forming a hydrogel above the gelation onset (Figure 2C and D). The UPy-UPy interactions are inherently dynamic, thus can dynamically associate and dissociate in the assembled fiber forming the crosslinks that build up the network (Figure 2C).

As stated above, under basic conditions, these cross-links are broken leading to disintegration of the transient network. To investigate this hypothesis in more detail, we studied the 


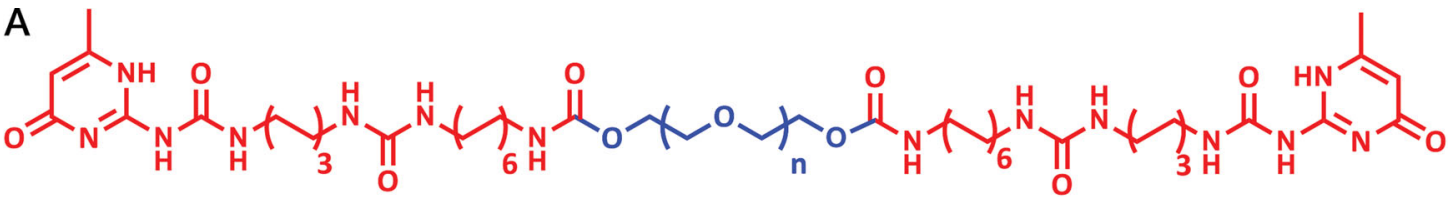
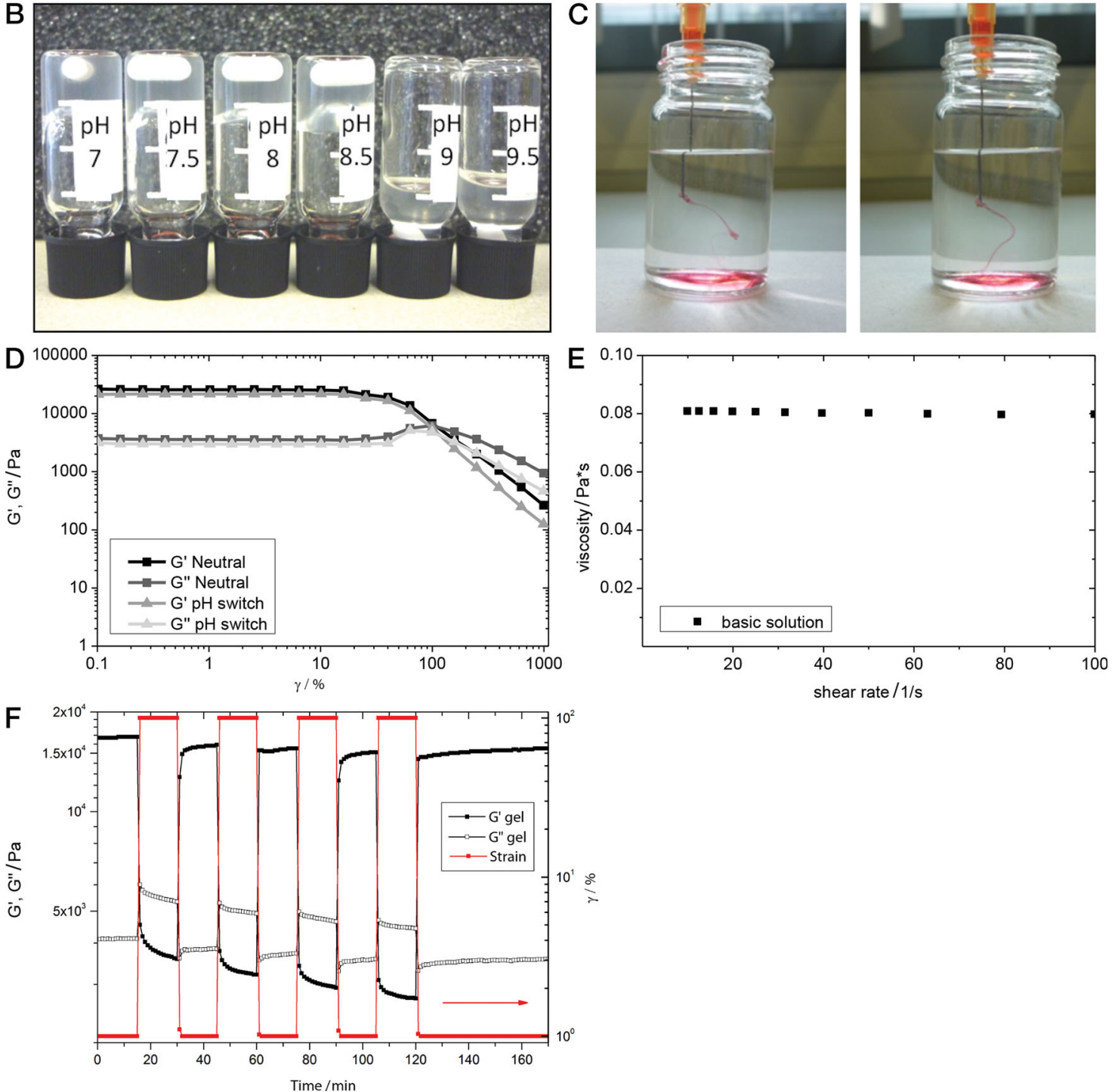

Figure 1. Structure, $\mathrm{pH}$ behavior, and rheological properties of UPy-hydrogelator polymers. A) Structure of the UPy-hydrogelators with the hydrophilic PEG block $\left(n=227 / 454, M_{n}=10 / 20 \mathrm{kDa}\right)$ in blue and the UPy-alkyl-urea end groups in red. B) Overview of the $\mathrm{pH}$-dependent behavior at a pH range from 7 to 9.5. C) Injection of a liquefied $10 \mathrm{wt} \%$ UPy-10k sample in PBS (colored in red) into a neutral PBS solution. Immediate gelation occurs when the basic solution comes in contact with the neutral solution. D) Rheology measurements of a $10 \mathrm{wt} \% \mathrm{UPy}-10 \mathrm{k}$ gel before and after the pH switch. E) Viscosity measurement of the liquefied $10 \mathrm{wt} \%$ UPy-10k gel matching the state upon catheter injection. F) Dynamic strain amplitude test of $10 \mathrm{wt} \%$ UPy-10k gel at $37^{\circ} \mathrm{C}$ showing the self-healing behavior over four cycles.

hydrogelator morphology at nanometer level using cryo-transmission electron microscopy (cryo-TEM), atomic force microscopy (AFM), dynamic light scattering (DLS), and Fourier-transformed infrared (FT-IR) spectroscopy both at neutral and basic conditions. Cryo-TEM shows the presence of rigid and elongated fibers in both neutral and basic solution, with average lengths of 75 and $104 \mathrm{~nm}$, respectively (Figure 2E and F, Supplementary
Information). This shows that a high $\mathrm{pH}$ does not influence the fiber formation. AFM imaging shows a hierarchical assembly of fibers for the polymers in water (Supplementary Information). In the basic $\mathrm{pH}$ sample, the assemblies seem more rigid and the variation in height is significantly less than in the neutral assembly, suggesting that the fibers are assembled out of fewer individual stacks. This indeed indicates a disrupted lateral 

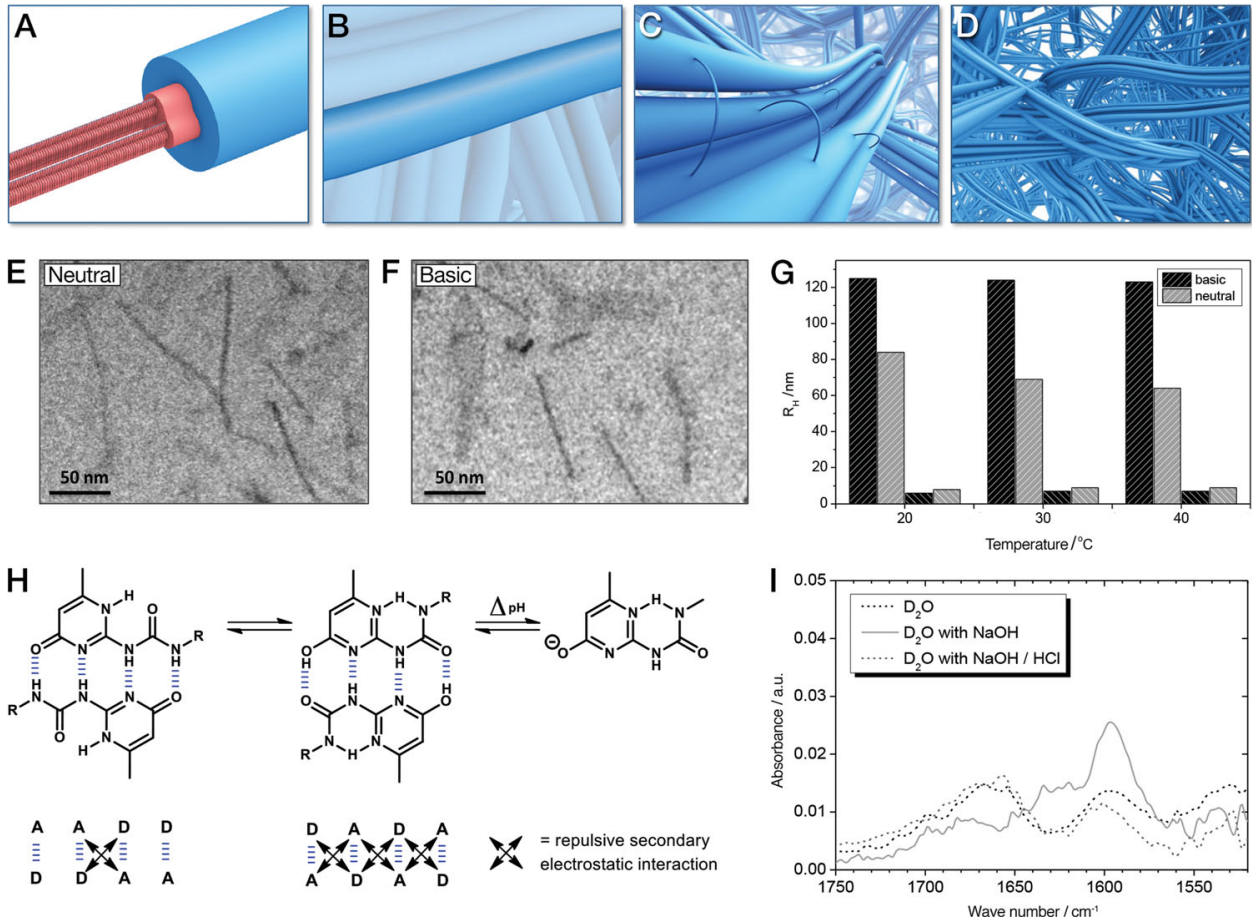

Figure 2. Structural analysis of UPy hydrogelators in neutral and basic environment. A-D) Proposed schematic molecular picture of the hydrogel material: zoom into the hydrogel fibers. A) Fibers are composed of multiple UPy-stacks (red), surrounded by a hydrophilic PEG corona (blue). B) Fibers can align and C) cross-link by UPy-polymers from one fiber into a neighboring fiber. D) UPy-fibers assemble into a transient network. E and F) Cryo-TEM of 1 wt\% solution of UPy-20k demonstrates the existence of elongated fibers in both E) neutral and F) basic environment. G) DLS on 1 wt\% UPy-20k solutions at various temperatures indicate the presence of two distinct populations in solution, micelles, and fibers, for both $\mathrm{pH}$ conditions. $\mathrm{H}$ ) UPytautomeric forms: the keto-tautomer and the enol-tautomer can form homodimers, whereas the enolate formed after deprotonation cannot dimerize. I) FT-IR on UPy-10k solutions of the $\mathrm{C}=\mathrm{O}$ stretch vibration indicating the existence of the enolate at basic conditions.

self-assembly due to loss of cross-linking in the basic state. Furthermore, DLS confirms the cryo-TEM measurements. DLS demonstrates the presence of two distinct distributions, which are proposed to be fibers and micelles, in both the neutral and basic $\mathrm{pH}$ environment (Figure 2G). The size dimensions calculated using the Stokes-Einstein equation to translate diffusion to hydrodynamic radius (with the assumption of spherical and noninteracting particles) match the cryo-TEM measurements. The breaking of the cross-links, and therefore the sol-gel transition, is proposed to be the result of deprotonation of the UPymoiety when present in the enol-tautomer, yielding the enolate (Figure 2H). FT-IR confirms this enolate-formation (Figure 2I). Modeling of the tautomeric states of the UPy-moiety in various $\mathrm{pH}$ environments shows a $\mathrm{p} K_{a}$ of the enol-OH of $\approx 7.2$, and the enolate anion being the dominant species present in a $\mathrm{pH}$ range from 8.5 to $12.5 .^{[35]}$ Upon dissociation, the UPy-group experiences a different environment than inside the fiber and a change in tautomeric form occurs, affecting the stability of the assembled system. The charged species cannot get incorporated back in the UPy-fiber to form a cross-link to stabilize the UPyhydrogel. The preservation of molecular ordered fibrillar assemblies forms the fundamental origin of this hydrogel material. As a second hierarchy in order, the switching between gel-like and solution-like material results from the spatiotemporal formation of $\mathrm{pH}$-sensitive interfiber cross-links. It is this combination of selective, hierarchical responsiveness that renders our material unique in its kind, and enables catheter injection to be explored for in vivo application.

\subsection{Biocompatibility In Vitro}

Cell compatibility is essential for the system to be applicable in.vivo. The hydrogelator formulation needs to be biocompatible both at neutral and basic $\mathrm{pH}$. The $\mathrm{pH}$ effect of different buffers on cardiomyocyte progenitor cells (CMPC) was screened, being milliQ, $0.9 \% \mathrm{NaCl}$, and PBS, all buffers at both neutral and basic $\mathrm{pH}$ (Supplementary Information). No toxicity was observed for the UPy-10k hydrogels prepared in different buffers at a physiological pH. For the elevated $\mathrm{pH} 9$ preparations, only the UPy-hydrogel based on PBS did not show any signs of cell toxicity. These in vitro studies show that the hydrogel system at both neutral (gel-state) and elevated $\mathrm{pH}$ (sol-state) in PBS is biocompatible. Additionally, biomaterials used for in vitro or in vivo applications need to be free of contaminating bacteria and endotoxins. To evaluate the presence of lipopolysaccharides present in our materials, an endotoxin test was performed. The concentration of endotoxin for all samples prepared in PBS was below the FDA approved endotoxin value of $0.5 \mathrm{EU} \mathrm{mL}^{-1}$ (Supplementary Information). 


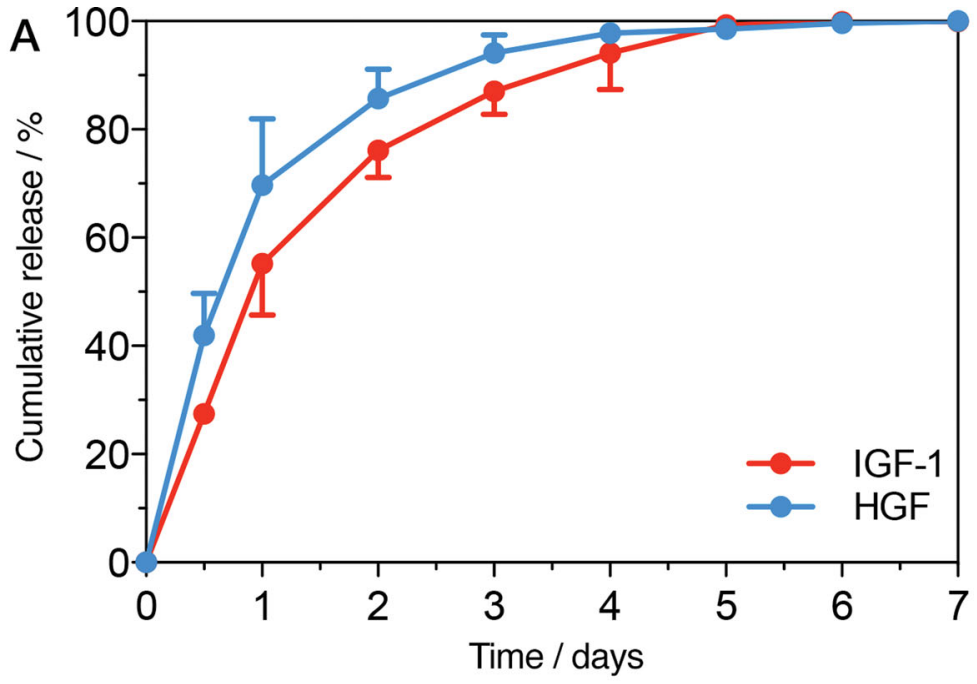

B

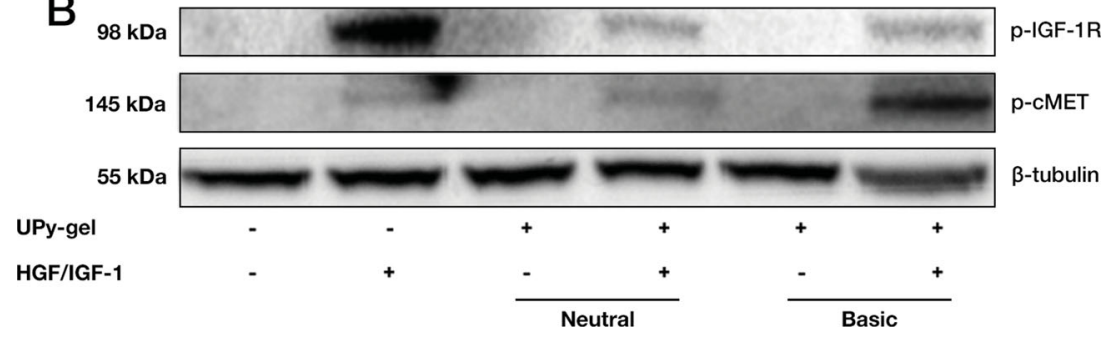

Figure 3. Release profile and bioactivity of HGF/IGF-1 embedded in the UPy-hydrogel. A) In vitro release characteristics of IGF-1 and HGF from the UPy-hydrogel by daily collection of medium at $37^{\circ} \mathrm{C}$. Both HGF and IGF-1 display an initial outburst (5 min after start of the experiment) followed by a 4-d release. B) Western blot analysis showing that CMPC express IGF-1R and CMET receptors that can be activated by IGF-1 (100 $\left.\mathrm{ng} \mathrm{mL}^{-1}\right)$ and HGF (100 $\mathrm{ng} \mathrm{mL}^{-1}$ ), respectively. At both $\mathrm{pH}$ levels, pristine UPy-hydrogel did not cause phosphorylation of IGF-1R ( - -IGF-1R) and CMET ( $\mathrm{p}-\mathrm{cMET}$ ). Medium collected from release studies of UPy-hydrogel loaded with HGF/IGF-1 show that the released HGF and IGF-1 can still activate their receptors, both in UPy-hydrogel with a $\mathrm{pH} 7.4$ (neutral) and the injectable $\mathrm{pH} 9$ (basic).

\subsection{Growth Factor Incorporation and Release}

Stimulation of endogenous cardiac repair through GF injection can benefit significantly from a sustained release over time instead of a single bolus injection. ${ }^{[36,37]}$ Data from different preclinical studies show the ability of selected GF, HGF and IGF-1, to enhance endogenous cardiac stem cell response to an ischemic site of injury. ${ }^{[38-40]}$ Here, both HGF and IGF-1 were incorporated in the UPy-10k hydrogel, and their release profile was subsequently studied in.vitro. A prolonged HGF and IGF-1 release from the UPy-hydrogel was achieved during $7 \mathrm{~d}$ (Figure 3A). The release of HGF was characterized by an initial outburst of $41 \%$, followed by a sustained release until $97 \%$ was released by the end of day 4. For IGF-1, 27\% was initially released whereas subsequent release showed similar release kinetics as $\mathrm{HGF}$, with a $94 \%$ release by day 4 . The difference in burst release between the different GF proteins might be attributed to the difference in size, which is $103 \mathrm{kDa}$ for HGF and $17 \mathrm{kDa}$ for IGF-1, respectively. Since PEG is nonfouling, we propose that there are no aspecific noncovalent interactions between the proteins and the hydrogel, suggesting that the difference in release can only be attributed to a size-dependent release profile. Following incubation in the basic UPy-hydrogel both IGF-1 and HGF showed preserved bioactivity shown by their affinity and ability to phosphorylate their corresponding receptors IGF-1R and cMET in CMPC (Figure 3B). Furthermore, after $7 \mathrm{~d}$ of in vitro incubation, the UPy-10k hydrogel was visually still present, indicating the stability of the hydrogel.

\subsection{Intramyocardial Delivery}

After the initial studies in,vitro, we assessed the feasibility of intramyocardial delivery in a porcine model of myocardial infarction. In total, four injections of $0.2 \mathrm{cc}$ were placed in one heart: two injections with SPIOS-labeled UPy-10k hydrogel in the anteroseptal wall of the left ventricle and two injections with UPy-hydrogel loaded with human recombinant HGF/IGF-1 in the lateral (infarcted) wall. These injections were guided to the borderzone of the infarction based on electromechanical mapping (EMM) (Figure 4A and B). Six hours after injection, the labeled UPy-hydrogel was successfully visualized by cardiac MRI (Figure 4A), and subsequently by histological analysis (Supplementary Information). With regard to safety issues, we observed no cardiac arrhythmias or tamponade within $6 \mathrm{~h}$ after the injection. Next, we assessed the GF distribution within the pig heart by quantifying the human-specific HGF and IGF-1 at the injection site in the infarct borderzone and in the opposite septal wall (i.e., remote area). Because of the crossreactivity with endogenous porcine HGF, only human specific IGF-1 was successfully identified at the site of injection (borderzone) and in the septal wall of the right ventricle (remote area) (Figure 4C and D). These data suggest an effective intramyocardial gradient of GF, increasing toward the borderzone of the infarction. Attraction of endogenous cardiac stem cells toward the infarct borderzone has shown to be beneficial for recovery in terms of improved blood flow to the infarcted area and formation of new viable cardiomyocytes. ${ }^{[38]}$ These in vivo data show feasibility of the UPy-hydrogel system as intramyocardial drug carrier and appears to be safe on short term follow-up, up to $6 \mathrm{~h}$.

\subsection{Growth Factor Induced Cardiac Repair and Adverse Remodeling}

The effect of UPy-hydrogel-mediated release on HGF/IGF-1 treatment in a porcine model of chronic ischemia was evaluated. Four weeks after an acute myocardial infarction, 10 NOGA guided injections were intramyocardially placed in 

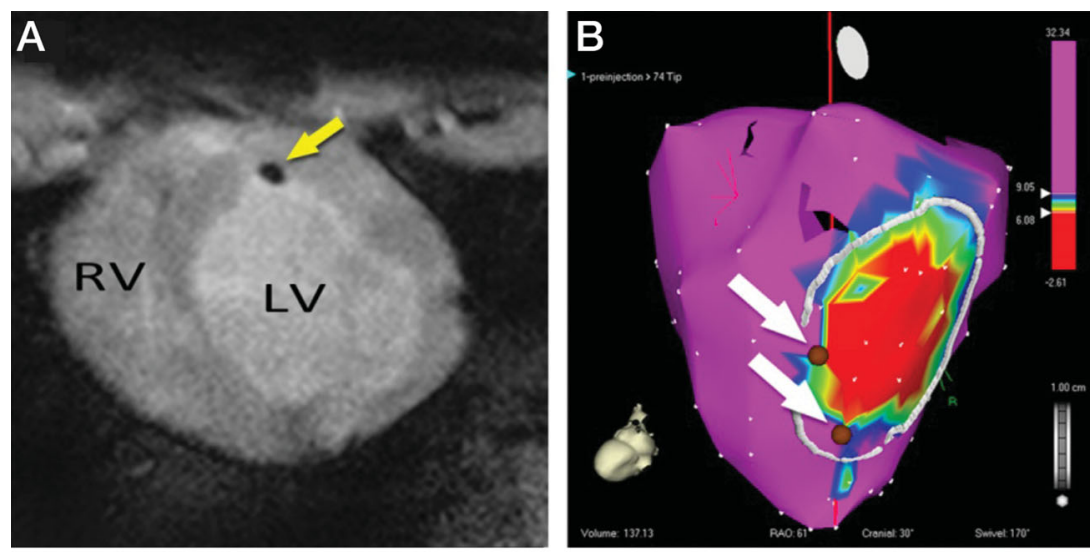

C

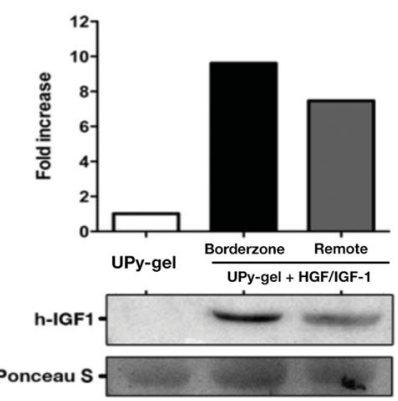

D
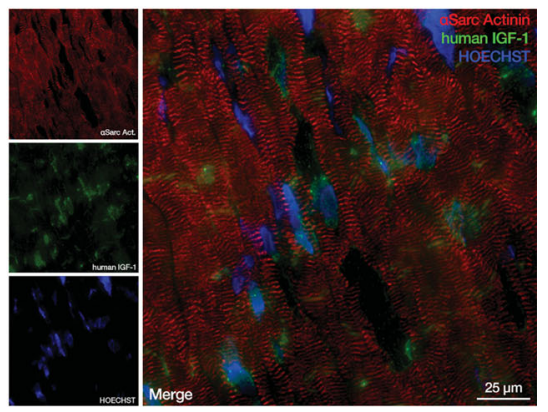

Figure 4. Transendocardial injection of UPy-hydrogel loaded with HGF/IGF-1. A) Cardiac MRI short axis plane showing the UP-gel containing SPIOS, visible as focal depressed T2* intensity, indicated by the yellow arrow. B) Electromechanical mapping of the left ventricle indicating the UPy-hydrogel + HGF/IGF-1 injections (white arrows) in the borderzone of the infarct (red signal, defined as $<6 \%$ on the local linear shortening map). C) Western blot analysis of human IGF-1 in the myocardial borderzone injected with pristine UPy-hydrogel, or UPy-hydrogel + HGF/ IGF-1 injected into the borderzone (BZ). After transendocardial injection, human IGF-1 was also detected in the opposing septal wall of the right ventricle (Remote). Ponceau S was used as a loading control to correct for differences in protein quantity. D) Immunofluorescent labeling of human IGF-1 (in green) shows cytoplasmatic and predominately perinuclear uptake by cardiomyocytes. Alfa-sarcomeric actin is shown in red. Nuclei are stained by HOECHST (blue).

\section{Conclusions}

Here we showed the development of a synthetic pH-switchable supramolecular hydrogel that is injectable through the long, narrow lumen of a state-of-the-art catheter mapping system. The catheter-injected hydrogelator solution transforms into a locally controlled drug release reservoir by immediate gelation upon contact with heart tissue and additionally is proposed to self-heal at the site of injection. The preservation of molecular ordered fibrillar assemblies forms the fundamental origin of this hydrogel material. As a second hierarchy in order, the switching between gel-like and solution-like material results from the spatiotemporal formation of $\mathrm{pH}$-sensitive interfiber cross-links. When loaded with the GF, HGF and IGF-1, we showed that the supramolecular hydrogelator system is able to reduce scar collagen in a chronic myocardial infarction pig model. To our knowledge, this is the first large animal study where NOGA catheter injection of a synthetic $\mathrm{pH}$-switchable hydrogel system loaded with GF for controlled release is reported. Because the NOGA ${ }^{\mathrm{TM}}$ catheter system is currently used in dedicated clinical centers, it will be together with our unique $\mathrm{pH}$-switchable hydrogel system eminently valuable for the development of a novel therapy for treatment of ischemic heart disease. ${ }^{[41,42]}$ the infarct borderzone. Three treatment groups were tested, consisting of the pristine UPy-10k hydrogel, HGF/IGF-1 in saline, and UPy-10k hydrogel loaded with HGF/IGF-1. Four weeks after injection, no adverse events (e.g., fever, cardiac arrhythmias, shortness of breath) were recorded during follow-up, and liver and renal functions were comparable in all groups (data not shown). The myocardial scar tissue was visualized by $1 \%$ triphenyl-tetrazolium chloride. All treatment groups macroscopically and histologically displayed roughly similar scar tissue formation in the lateral wall of the left ventricle (Figure 5A-C and 5G-I). However, after quantification of the Picrosirius Red stained tissue slices (Figure 5D-F and 5J-L) it was shown that the collagen content of the infarct scar in the UPy-hydrogel loaded with HGF/ IGF-1-treated animals was reduced $(12.9 \pm 3.1 \%)$ compared with the pristine UPy-hydrogel $(25.2 \pm 5.7 \%$; $P=0.02)$ (Figure $5 \mathrm{P})$. Remarkably, patched-like clusters of viable myocardium were present dispersed in between the dense collagen fibers in the UPy-hydrogel-loaded HGF/IGF-1 group (Figure 5I). These results suggest a more favorable remodeling process in the UPy-hydrogel loaded with HGF/IGF-1 than in soluble HGF/IGF-1-treated animals.

\section{Experimental Section}

Preparation of UPY-Hydrogels: The UPy-hydrogelators with $\bar{M}_{\mathrm{n}, \mathrm{PEG}}=10 \mathrm{~kg} \mathrm{~mol}^{-1}$ or $\bar{M}_{\mathrm{n}, \mathrm{PEG}}=20 \mathrm{~kg} \mathrm{~mol}^{-1}$, i.e., UPy-10k and UPy- $20 \mathrm{k}$, respectively, were synthesized by SyMO-Chem BV, Eindhoven, The Netherlands. ${ }^{[31]}$ For solution studies, i.e., using concentrations of $1 \mathrm{wt} \%$, the PEG-20k hydrogel was used since the shorter hydrophilic domain of the PEG-10k materials causes precipitation in time. For the gel and biomedical experiments, the higher stability of the PEG-10k is preferred considering the resulting prolonged lifetime of the gel.

Growth Factors and UPY-Hydrogels: For the preparation of the hydrogels, polymer solutions were prepared by dissolving $10 \mathrm{wt} \%$ in PBS $\mathrm{pH} 7.4$ by stirring at $70{ }^{\circ} \mathrm{C}$ for $2 \mathrm{~h}$ and subsequently cooled to RT. To liquefy the polymer solution, the $\mathrm{pH}$ was raised by adding $2 \mu \mathrm{L}$ aliquots of a $0.1 \mathrm{M} \mathrm{NaOH}$ stock solution. Human recombinant IGF-1 (Miltenyi Biotec) and human recombinant HGF (Miltenyi Biotec) were mixed in by slow stirring for 10 min yielding a final concentration of $500 \mathrm{ng} \mathrm{mL}^{-1}$ of each GF. For noninvasive assessment of the UPy-hydrogel by cardiac MRI in,vivo, SPIOS were mixed with UPy-hydrogel $\mathrm{pH} 9$ by gentle stirring at room temperature for 3 min yielding a final concentration of $13.6 \mu \mathrm{g} \mathrm{mL} \mathrm{m}^{-1}$. Solutions were then UV-sterilized for at least $1 \mathrm{~h}$ before use.

Rheology Experiments on Gel Samples: Oscillatory rheology experiments were performed on an Anton Paar Physica MCR 501 rheometer. Mechanical properties of the viscoelastic hydrogel material under neutral or neutralized conditions were assayed using a parallel 
UPy-ge
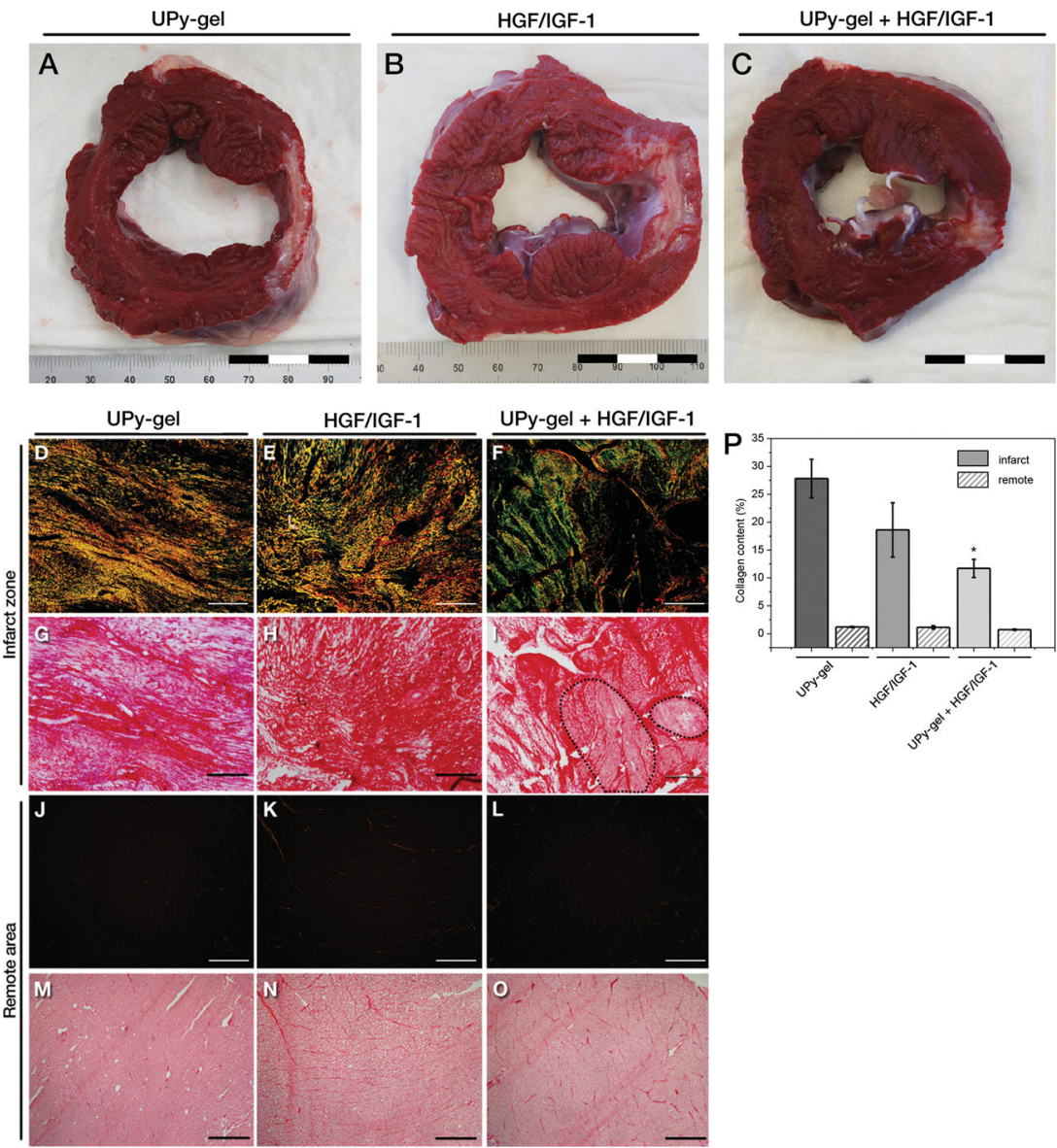

UPy-gel + HGF/IGF-1

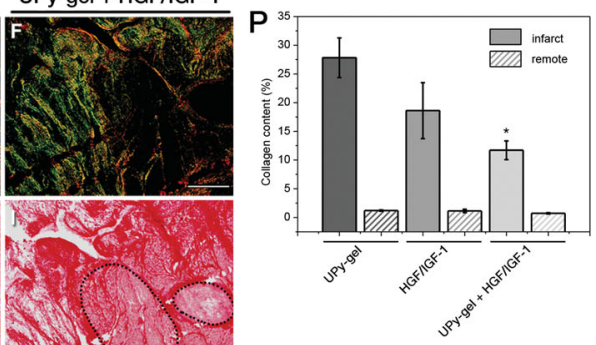

Figure 5. Adverse remodeling and GF induced cardiac repair. A-C) The infarct scar is visible as a white color stained with $1 \%$ triphenyl-tetrazolium chloride in all three treatment groups; A) pristine UPy-hydrogel, B) HGF/IGF-1, and C) UPy-hydrogel + HGF/IGF-1. A-C) Scale bars represent $3.0 \mathrm{~cm}$. D-F/J-L) Cryosections were stained with Picrosirius Red to quantify collagen deposition using polarized light on the infarct zone D-F) and the remote area showing viable myocardium with scarcely dispersed collagen J-L). G-I/M-O) The bright field images of the Picrosirius Red staining. I) Patches of cardiomyocytes were observed within the infarct zone in the UPy-hydrogel + HGF/IGF-1-treated group (demarcated areas). P) Quantification of total collagen content per field demonstrates reduced collagen content in the infarct scar in the UPy-hydrogel + HGF/IGF-1 compared with the UPy-hydrogel ( $*$ denotes $P=0.02$ ). The data are expressed as mean \pm SEM. Differences in data were evaluated with a one-way analysis of variance (ANOVA) followed by Tukey post-hoc analysis. D-O) Scale bars represent $500 \mu \mathrm{m}$.

(PP 25)-plate geometry. The linear viscoelastic regime of the samples was obtained from amplitude sweep experiments that were from $0.1 \%$ to $1000 \%$ strain at $1 \mathrm{rad} \mathrm{s}^{-1}$ angular frequency. Frequency sweep measurements were recorded from 0.1 to $100 \mathrm{rad} \mathrm{s}^{-1}$ at $1 \%$ strain. Measurements were performed at $20{ }^{\circ} \mathrm{C}$ unless stated otherwise.

Viscosity Measurements on Basic Solution: Steady-state viscosity measurements were performed using a cone (CP-25-1) -plate geometry with a $1^{\circ}$ angle. UPy-10k was dissolved in PBS buffer $\left(\mathrm{pH} \mathrm{7.4)}\right.$ at $70{ }^{\circ} \mathrm{C}$, cooled to room temperature and transferred to the injectable fluid state by addition of a small aliquot of $\mathrm{NaOH}$, yielding a $10 \mathrm{wt} \%$ hydrogel. The viscosity as a function of shear rate $(\dot{\gamma})$ was measured from 0.1 to 100 rad $\mathrm{s}^{-1}$ at $20^{\circ} \mathrm{C}$. The zero-shear viscosity, $\eta_{0}$, was extrapolated from the Newtonian plateau to the $\gamma$-axis.

Cryogenic Transmission Electron Microscopy: Sample preparation was performed by pipette addition of $1 \mathrm{wt} \%$ UPy-20k solutions ( $3 \mu \mathrm{L}$; neutral and basic conditions) onto holey carbon film (hole diameter $=2 \mu \mathrm{m}$ ) supported by a copper TEM grid before manual blotting at $100 \%$ relative humidity and $21{ }^{\circ} \mathrm{C}$. Immediate vitrification was performed by automated
www.MaterialsViews.com

plunging of the grid into liquid ethane. Upon completion of the set time, automated vitrification was performed. Samples were stored under liquid nitrogen before imaging. Cryo-TEM was performed on a FEI Tecnai 20 (type Sphera) TEM operating with a $200 \mathrm{kV}$ LaB6 filament and a bottom mounted $1024 \times 1024$ Gatan msc $794^{\mathrm{TM}}$ CCD camera.

Dynamic Light Scattering: DLS experiments on a 1 wt\% UPy-20k solution under neutral and basic conditions were conducted on an ALV/CGS-3 MD-4 compact goniometer system equipped with a multiple tau digital real-time correlator (ALV-7004) (solid state laser: $\lambda=532 \mathrm{~nm} ; 40 \mathrm{~mW}$ ). Typical experiments covered a scattering angle from $20^{\circ}$ to $150^{\circ}$, averaging over $5 \times 30 \mathrm{~s}$ runs at a temperature of $20-40{ }^{\circ} \mathrm{C}$ in $10^{\circ} \mathrm{C}$ intervals. Data were processed using after ALV freeware and scattering artifacts were excluded from the analysis. The hydrodynamic radius $\left(R_{\mathrm{h}}\right)$ was extracted from the measured diffusion coefficient $D_{0}=\Gamma(x) / q^{2}$ assuming validity of the Stokes-Einstein relation, $R_{\mathrm{h}}=k T / 6 \pi \eta D$ ( $k$ being the Boltzmann constant and $\eta$ the solvent viscosity) for spherical objects.

Infrared Spectroscopy: UPy-10k samples (neutral and basic conditions) were prepared with a total concentration of $1 \mathrm{wt} \%$ in $\mathrm{D}_{2} \mathrm{O}$. Solution infrared spectra were recorded on a PerkinElmer spectrum One FT-IR spectrometer and measured at a resolution of $4 \mathrm{~cm}^{-1}$, by coadding 128 scans. Samples were loaded in a fixed path length $(50 \mu \mathrm{m})$ cell with $\mathrm{CaF}_{2}$ windows, and used immediately for IR measurements.

Cell Culture Experiments and Biocompatibility Testing: CMPC derived from human fetal hearts were isolated as described. ${ }^{[43]}$ Briefly, human fetal hearts were collected following elective abortion, after written informed consent was provided. The protocol was approved by the Medical Ethics committee of the University Medical Center Utrecht and is in line with the principles outlined in the Declaration of Helsinki. Briefly, the CMPC were isolated by magnetic bead sorting using Sca-1 coupled beads (Milteny). CMPC were then maintained in growth medium and used for different assays. The toxicity of UPy-10k hydrogel formulations on CMPC was tested in vitro using a lactate dehydrogenase (LDH) assay (SigmaAldrich). CMPC were cultured in M-199 medium (Sigma-Aldrich) with 2\% FBS (Invitrogen) and incubated with $100 \mu \mathrm{L}$ of $10 \mathrm{wt} \%$ UPy-hydrogel based on milliQ, $\mathrm{NaCl} 0.9$ or PBS (all buffers were tested both at $\mathrm{pH} 7.4$ and $\mathrm{pH}$ 9.0). After $3 \mathrm{~d}$ of incubation, medium was collected and tested for the presence of LDH as a marker for cell damage according to manufacturer's instruction.

Growth Factor Release and Bioactivity In Vitro: Human recombinant HGF and human recombinant IGF-1 dissolved in milliQ water were added to liquefied UPy-10K hydrogel in PBS $\mathrm{pH} 9$ yielding a final concentration of $500 \mathrm{ng} \mathrm{mL} \mathrm{m}^{-1}$ of each GF. $100 \mu \mathrm{L}$ of UPy-hydrogel with GF was incubated in $200 \mu \mathrm{L}$ of medium in 48-well plates on a rotational shaker $(90 \mathrm{rpm})$ at $37{ }^{\circ} \mathrm{C}$ for $7 \mathrm{~d}$. $75 \mu \mathrm{L}$ of medium was removed daily and replaced with fresh medium. The collected medium was quantified for HGF and IGF-1 using ELISA detection (R\&D Systems). The bioactivity of the GF released was investigated as follows: CMPC were subjected to serum starvation for $18 \mathrm{~h}$, followed by supplementation of medium collected from the HGF/IGF-1 release study for $15 \mathrm{~min}$. Cells were lysed and Western blot analysis was performed for phosphorylated CMET for HGF (Cell Signaling) and phosphorylated IGF receptor 1 (pIGF-R1) for IGF-1 (Cell Signaling). IGF-1 (100 ng mL $\mathrm{mL}^{-1}$ ) and HGF (100 ng mL $\mathrm{m}^{-1}$ ) dissolved in MilliQ water served as a positive control. 
Animal Experiments and Study Design: In total thirteen pigs (female Dutch landrace, weighing approximately $70 \mathrm{~kg}$ ) received humane care in compliance with the national guidelines on animal care and prior approval by the Animal Experimentation Committee of the Faculty of Medicine, Utrecht University, The Netherlands. Myocardial infarction was induced by $75 \mathrm{~min}$ of intracoronary balloon occlusion of the proximal left circumflex artery. Four weeks later, the animals underwent 3D EMM of the left ventricle for infarct and borderzone localization using the NOGA ${ }^{\mathrm{TM}}$ catheter system (Biosense Webster, Cordis, Johnson \& Johnson, USA). Intramyocardial delivery was performed using the NOGA $^{\mathrm{TM}}$ Myostar system (Biosense Webster, Cordis, Johnson \& Johnson).

Short-Term In Vivo Tracking of Growth Factor Distribution: Regarding the in vivo tracking of UPy-hydrogel, four injections of $0.2 \mathrm{cc}$ were placed, two injections with SPIOS-labeled UPy-10k hydrogel in the anteroseptal wall of the left ventricle and two injections with HGF/IGF-1-loaded UPy-hydrogel to the borderzone of the infarction (lateral wall). Six hours after injection, two animals underwent in vivo tracking of the hydrogel by cardiac MRI. Tissue samples collected from the septal wall and the injection site were snap frozen for western blot analysis for human IGF-1 (Abcam; dilution 1:1000) and immunofluorescent staining for human anti-IGF-1 (Abcam; dilution 1:100) and for alfa-sarcomeric actin (Sigma, dilution 1:50).

The Effect of Sustained Release on Cardiac Function: Four weeks after myocardial infarction induction 10 animals were injected with pristine UPy-10k hydrogel $(n=3)$, saline dissolved HGF/IGF-1 (both $\left.500 \mathrm{ng} \mathrm{mL}^{-1}\right)(n=3)$ and UPy-hydrogel loaded with HGF/IGF-1 (both $\left.500 \mathrm{ng} \mathrm{mL} \mathrm{L}^{-1}\right)(n=4)$. Then, the animals were followed for 4 weeks, and subsequently euthanized by exsanguination under general anesthesia. After excision of the heart, the heart was cut into five slices from base to apex and infarct size was determined by $1 \%$ triphenyl-tetrazolium chloride staining. Tissue samples collected of the septal wall (remote) and the infarction zone were snap frozen for histological analysis. Cryosections $(7 \mu \mathrm{m})$ were prepared on a microtome (Leika) and fixed in $4 \%$ formalin for $10 \mathrm{~min}$. Next, the sections were incubated in $0.1 \%$ Sirius red (BDH) in picric acid (Sigma) for $8 \mathrm{~min}$ at room temperature. After counterstaining the nuclei with hematoxylin for $1 \mathrm{~min}$, the sections were dehydrated, cleared, and mounted. The quantification was performed on 30 fields per animal of UV polarized light on a microscope (Olympos DP71) at 40x magnification and automatically quantified for the percentage of collagen per field using Image) software for Macintosh (version $1.44 \mathrm{~g}$ ). The analysis was performed by an investigator blinded to the treatment allocation.

Statistical Analysis: The data are expressed as mean \pm SEM. Differences in data were evaluated with a one-way ANOVA followed by Tukey post-hoc analysis. Data analysis was performed on SPSS v19.0.0 software for Macintosh. Probabilities of $P<0.05$ were considered to be statistically significant; $P<0.05$ is depicted as $*$

\section{Supporting Information}

Supporting Information is available from the Wiley Online Library or from the author.

\section{Acknowledgements}

The authors thank Henk Janssen and Joris Peters for the supply of UPy-polymers. Lenny Meijer is acknowledged for performing the endotoxin tests. Pol Besenius is thanked for performing Cryo-TEM analysis. Ilja Voets is acknowledged for assistance with light scattering analysis. Cees Verlaan, Marlijn Jansen, Joyce Visser, Merel Schurink, and Maringa Emons are acknowledged for their excellent technical assistance with the animal experiments. Koen Pieterse from the ICMS Animation studio is acknowledged for the production of the schematic representations. MMCB is funded by a NWO Toptalent Fellowship. SK is funded by the HGG group B.V. and the Wijnand M. Pon Stichting. YN is supported by a grant from the Dutch government to the Netherlands
Institute for Regenerative Medicine (NIRM). EWM is supported by the European Research Council (ERC). PYWD is supported by the Council for Chemical Sciences of the Netherlands Organization for Scientific Research (CW-NWO) and the European Research Council (ERC). This research forms part of the Project P1.04 SMARTCARE of the BioMedical Materials institute, cofunded by the Dutch Ministry of Economic Affairs, Agriculture and Innovation. The financial contribution of the Nederlandse Hartstichting is gratefully acknowledged (DF and FvS). The research leading to these results has received funding from the Dutch Ministry of Education, Culture and Science (Gravity program 024.001.035) (ACHP, EWM, PYWD).

Received: February 27, 2013

Revised: March 27, 2013

Published online: June 21, 2013

[1] D. Lloyd-Jones, R. J. Adams, T. M. Brown, M. Carnethon, S. Dai, G. De Simone, T. B. Ferguson, E. Ford, K. Furie, C. Gillespie, A. Go, K. Greenlund, N. Haase, S. Hailpern, P. M. Ho, V. Howard, B. Kissela, S. Kittner, D. Lackland, L. Lisabeth, A. Marelli, M. M. McDermott, J. Meigs, D. Mozaffarian, M. Mussolino, G. Nichol, V. L. Roger, W. Rosamond, R. Sacco, P. Sorlie, R. Stafford, T. Thom, S. Wasserthiel-Smoller, N. D. Wong, J. Wylie-Rosett, Circulation 2010, 121, 948.

[2] K. Krause, K. Jaquet, C. Schneider, S. Haupt, M. V. Lioznov, K. M. Otte, K. H. Kuck, Heart 2009, 95, 1145

[3] R. J. Laham, M. Rezaee, M. Post, F. W. Sellke, R. A. Braeckman, D. Hung, M. Simons, Drug Metab. Dispos. 1999, $27,821$.

[4] J. Leor, Y. Amsalem, S. Cohen, Pharmacol. Ther. 2005, 105, 151.

[5] G. C. Engelmayr Jr., M. Cheng, C. J. Bettinger, J. T. Borenstein, R. Langer, L. E. Freed, Nat. Mater. 2008, 7, 1003.

[6] H. Piao, J. S. Kwon, S. Piao, J. H. Sohn, Y. S. Lee, J. W. Bae, K. K. Hwang, D. W. Kim, O. Jeon, B. S. Kim, Y. B. Park, M. C. Cho, Biomaterials 2007, 28, 641 .

[7] R. C. Scott, D. Crabbe, B. Krynska, R. Ansari, M. F. Kiani, Expert Opin. Drug Deliv. 2008, 5, 459.

[8] L. Gepstein, G. Hayam, S. A. Ben-Haim, Circulation 1997, 95, 1611.

[9] K. L. Christman, H. H. Fok, R. E. Sievers, Q. Fang, R. J. Lee, Tissue Eng 2004, 10, 403.

[10] W. Dai, L. E. Wold, J. S. Dow, R. A. Kloner, J. Am. Coll. Cardiol. 2005, 46, 714.

[11] N. Landa, L. Miller, M. S. Feinberg, R. Holbova, M. Shachar, I. Freeman, S. Cohen, J. Leor, Circulation 2008, 117, 1388.

[12] T. Kofidis, D. R. Lebl, E. C. Martinez, G. Hoyt, M. Tanaka, R. C. Robbins, Circulation 2005, 112, 1173.

[13] J. L. Ifkovits, E. Tous, M. Minakawa, M. Morita, J. D. Robb, K. J. Koomalsingh, J. H. Gorman, 3rd, R. C. Gorman, J. A. Burdick, Proc. Natl. Acad. Sci. USA 2010, 107, 11507.

[14] W. N. Lu, S. H. Lu, H. B. Wang, D. X. Li, C. M. Duan, Z. Q. Liu, T. Hao, W. J. He, B. Xu, Q. Fu, Y. C. Song, X. H. Xie, C. Y. Wang, Tissue Eng. Part A 2009, 15, 1437.

[15] J. M. Singelyn, P. Sundaramurthy, T. D. Johnson, P. J. Schup-Magoffin, D. P. Hu, D. M. Faulk, J. Wang, K. M. Mayle, K. Bartels, M. Salvatore, A. M. Kinsey, A. N. Demaria, N. Dib, K. L. Christman, J. Am. Coll. Cardiol. 2012, 59, 751.

[16] N. A. Peppas, J. Z. Hilt, A. Khademhosseini, R. Langer, Adv. Mater. 2006, 18, 1345

[17] M. E. Davis, J. P. Motion, D. A. Narmoneva, T. Takahashi, D. Hakuno, R. D. Kamm, S. Zhang, R. T. Lee, Circulation 2005, 111, 442.

[18] X. Zheng Shu, Y. Liu, F. S. Palumbo, Y. Luo, G. D. Prestwich, Biomaterials 2004, 25, 1339 .

[19] D. A. Wang, C. G. Williams, Q. Li, B. Sharma, J. H. Elisseeff, Biomaterials 2003, 24, 3969.

[20] J. Li, X. Ni, K. W. Leong, J. Biomed. Mater. Res. 2003, 65, 196. 
[21] B. H. Lee, B. Vernon, Macromol. Biosci. 2005, 5, 629.

[22] N. Peppas, Hydrogels in Medicine and Pharmacy, CRC, Boca Raton, 1987.

[23] J. D. Hartgerink, E. Beniash, S. I. Stupp, Science 2001, 294, 1684.

[24] Q. Wang, J. L. Mynar, M. Yoshida, E. Lee, M. Lee, K. Okuro, K. Kinbara, T. Aida, Nature 2010, 463, 339.

[25] H. Yokoi, T. Kinoshita, S. Zhang, Proc. Natl. Acad. Sci. USA 2005, $102,8414$.

[26] S. Zhang, M. A. Greenfield, A. Mata, L. C. Palmer, R. Bitton, J. R. Mantei, C. Aparicio, M. O. de la Cruz, S. I. Stupp, Nat. Mater. 2010, 9, 594

[27] M. Zhou, A. M. Smith, A. K. Das, N. W. Hodson, R. F. Collins, R. V. Ulijn, J. E. Gough, Biomaterials 2009, 30, 2523.

[28] F. Z. Y. Gao, Q. Wang, Y. Zhanga, B. Xu, Chem. Soc. Rev. 2010, 39, 3425.

[29] G. L. Z. Yang, B. Xu, Acc. Chem. Res. 2008, 41, 315.

[30] R. P. Sijbesma, F. H. Beijer, L. Brunsveld, B. J. Folmer, J. H. Hirschberg, R. F. Lange, J. K. Lowe, E. W. Meijer, Science 1997, 278, 1601

[31] P. Y. Dankers, T. M. Hermans, T. W. Baughman, Y. Kamikawa, R. E. Kieltyka, M. M. Bastings, H. M. Janssen, N. A. Sommerdijk, A. Larsen, M. J. van Luyn, A. W. Bosman, E. R. Popa, G. Fytas, E. W. Meijer, Adv. Mater. 2012, 24, 2703.

[32] P. Y. Dankers, M. J. van Luyn, A. Huizinga-van der Vlag, G. M. van Gemert, A. H. Petersen, E. W. Meijer, H. M. Janssen, A. W. Bosman, E. R. Popa, Biomaterials 2012, 33, 5144.

[33] B. Bhana, R. K. Iyer, W. L. Chen, R. Zhao, K. L. Sider, M. Likhitpanichkul, C. A. Simmons, M. Radisic, Biotechnol. Bioeng. 2010, 105, 1148.

[34] P. Gonzalez-Tello, F. Camacho, E. Jurado, M. P. Paez, E. M. Guadix, Biotechnol. Bioeng. 1994, 44, 523.

[35] Marvin freeware online was used to plot the tautomeric distribution of UPy-species at various $\mathrm{pH}$ showing that the enolate forms the most abundant species at a $\mathrm{pH}>8.5$.
[36] E. Ruvinov, J. Leor, S. Cohen, Biomaterials 2010, 32, 565

[37] M. E. Davis, P. C. H. Hsieh, T. Takahashi, Q. Song, S. Zhang, R. D. Kamm, A. J. Grodzinsky, P. Anversa, R. T. Lee, Proc. Natl. Acad. Sci. USA 2006, 103, 8155.

[38] G. M. Ellison, D. Torella, S. Dellegrottaglie, C. Perez-Martinez, A. Perez de Prado, C. Vicinanza, S. Purushothaman, V. Galuppo, C. Iaconetti, C. D. Waring, A. Smith, M. Torella, C. Cuellas Ramon, J. M. Gonzalo-Orden, V. Agosti, C. Indolfi, M. Galiñanes, F. Fernandez-Vazquez, B. Nadal-Ginard, J. Am. Coll. Cardiol. 2011, $58,977$.

[39] A. Linke, P. Müller, D. Nurzynska, C. Casarsa, D. Torella, A. Nascimbene, C. Castaldo, S. Cascapera, M. Böhm, F. Quaini, K. Urbanek, A. Leri, T. H. Hintze, J. Kajstura, P. Anversa, Proc. Natl. Acad. Sci. USA 2005, 102, 8966.

[40] K. Urbanek, M. Rota, S. Cascapera, C. Bearzi, A. Nascimbene, A. De Angelis, T. Hosoda, S. Chimenti, M. Baker, F. Limana, D. Nurzynska, D. Torella, F. Rotatori, R. Rastaldo, E. Musso, F. Quaini, A. Leri, J. Kajstura, P. Anversa, Circ. Res. 2005, 97, 663.

[41] J. van Ramshorst, J. J. Bax, S. L. M. Beeres, P. Dibbets-Schneider, S. D. Roes, M. P. M. Stokkel, A. de Roos, W. E. Fibbe, J. J. Zwaginga, E. Boersma, M. J. Schalij, D. E. Atsma, JAMA 2009, 301, 1997.

[42] E. C. Perin, J. T. Willerson, C. J. Pepine, T. D. Henry, S. G. Ellis, D. X. M. Zhao, G. V. Silva, D. Lai, J. D. Thomas, M. W. Kronenberg, A. D. Martin, R. D. Anderson, J. H. Traverse, M. S. Penn, S. Anwaruddin, A. K. Hatzopoulos, A. P. Gee, D. A. Taylor, C. R. Cogle, D. Smith, L. Westbrook, J. Chen, E. Handberg, R. E. Olson, C. Geither, S. Bowman, J. Francescon, S. Baraniuk, L. B. Piller, L. M. Simpson, C. Loghin, D. Aguilar, S. Richman, C. Zierold, J. Bettencourt, S. L. Sayre, R. W. Vojvodic, S. I. Skarlatos, D. J. Gordon, R. F. Ebert, M. Kwak, L. A. Moye, R. D. Simari, JAMA 2012, 307, 1717.

[43] A. M. Smits, P. van Vliet, C. H. Metz, T. Korfage, J. P. Sluijter, P. A. Doevendans, M.-J. Goumans, Nat. Protoc. 2009, 4, 232. 